\title{
Towards an Ergonomics of Knowledge Systems: Improving the Design of Technology Enhanced Learning
}

\author{
David E. Millard ${ }^{1}$ and Yvonne Howard ${ }^{1}$, \\ ${ }^{1}$ Learning Societies Lab, School of Electronics and Computer Science, University of \\ Southampton, UK \\ \{dem,ymh\}@ecs.soton.ac.uk
}

\begin{abstract}
As Technology Enhanced Learning (TEL) systems become more essential to education there is an increasing need for their creators to reduce risk and to design for success. We argue that by taking an ergonomic perspective it is possible to better understand why TEL systems succeed or fail, as it becomes possible to analyze how well they are aligned with their users and environment. We present three TEL case studies that demonstrate these ideas, and show how an ergonomic analysis can help frame the problems faced in a useful way. In particular we propose using a variant of ergonomics that emphasizes the expression, communication and use of knowledge within the system; we call this approach Knowledge System Ergonomics.
\end{abstract}

Keywords: Design Methodologies, Ergonomics, Knowledge Systems

\section{Introduction}

Successful e-learning systems are difficult to design and create. In addition to traditional software engineering problems such as performance, openness, efficiency and interface design there is also a need to take a pedagogical view on the activities and processes enshrined in the software [1], to consider how the system fits into an existing complex ecosystem of physical and digital systems, and to take account of how it is affected by institutional policy and personal preferences [2].

These pressures can create highly specialized software systems that are difficult to transplant to other institutions or contexts, and are extremely brittle in the face of change. If we believe that more specialized pedagogically-informed systems are needed in education then helping technologists build systems that are a good fit to teachers and learners is a critical goal. In our view this can be described as a need for better Ergonomic Design, and we believe that taking this wider perspective is helpful in formulating new design principles for Technology Enhanced Learning (TEL) that will reduce risk and help TEL creators design for success.

In particular, through three different case studies, we suggest that since TEL systems can be seen as a particular kind of Knowledge System a variant of ergonomics focused on the knowledge models and knowledge processes within them can offer valuable insights into how to align a new TEL system with its users. We call this approach Knowledge System Ergonomics. 


\section{Background}

Usability has long been identified as a critical success factor for TEL [3]. Usability pioneers identify a number of key issues for design that apply to e-learning such as matching a system to the real world (using common language and terms), and user control and freedom [4]. Later researchers make the connection between usability and pedagogical considerations, introducing 'learnability' as a TEL design factor [5].

Ergonomics is the study of how to fit a system to a person, and although it is more traditionally thought of in the sense of physical devices and machines, it can equally be applied to digital systems and applications [6]. The environmental considerations of TEL fit well with principles of macro-ergonomics where a more holistic approach is taken to designing new systems or processes [7]. When applied in HumanComputer Interaction there tends to be an emphasis on information and cognitive tasks and so this type of approach is referred to as cognitive ergonomics [8]. More recently researchers have begun to explore if ergonomics can contribute to education, arguing for an ergonomic perspective on learning, directed at design improvements of physical learning environments [9]; for example, Rudolf et al. demonstrates that improved classroom ergonomics can improve student comfort and productivity [10].

We propose that we gain insight into the design of TEL systems by viewing them as knowledge systems. Definitions of knowledge in the classical world emphasized truth and belief, however with the rise of the Semantic Web and Linked Data broader definitions have become popular that focus on knowledge as information that has a "practical use in action" [11]. This is the perspective that we take: that knowledge is information that it is in an appropriate form for it to be applied to some task.

In this paper we argue that if we see e-learning applications as a form of knowledge system, the problem of designing TEL can be seen as one of Knowledge System Ergonomics, a combination of macro and cognitive ergonomics that could help designers create systems that are better aligned with users and usage contexts.

\section{Case Study 1: E-Learning Repositories (EdShare)}

Description: Over the last decade there has been tremendous growth in the interest in e-learning repositories. The Language Box and EdShare projects at the University of Southampton started in 2007, their objective was to learn from Web 2.0 sites and create a repository for the Language Teaching community, and another as the institutional repository for the University of Southampton [12]. Both projects took a participatory design approach coupled with agile development.

Difficulties and Challenges: Very early it became clear that there was a major disconnect between the needs of potential users, and the sorts of complex meta-data represented by Learning Objects; for example, users complained that their were too many fields and that the pedagogical terms didn't match to everyday reality [13]. We also struggled to get users to reuse materials in the repository. Initially we allowed users to put two types of item in the repository: Resources (that represented a raw set of materials that could be used for teaching) and Activities (that described what you might do with those materials). However, we found that teachers struggled to 
understand the difference between these types. In the end we scrapped Activities and instead introduced the notion of Remixing a Resource, this clones a Resource and allows the user to change the copy. This simple remix function was understood much more readily [14]. However, the biggest challenge was in getting users to engage with the repository. A major challenge was to understand why people were prepared to use sharing sites but not put their teaching materials into a traditional repository.

Ergonomic Perspective: In the case of Metadata the problem was of terminology and cognitive overload; because of the number of fields, and the unfamiliarity of their names, users were not prepared to spend time uploading content. By designing for minimal manual meta-data, and maximum automatic metadata, and by using everyday practical language we were able to massively reduce the barriers to use.

In the case of Reuse and Remixing, the problem was the sophistication of the information structures, and the way in which they required users to be able to abstract their teaching activities from their teaching content. This type of information abstraction is a key skill for software engineers, but it is alien to most users.

For engagement we found it useful to take a cognitive ergonomics perspective, and to look at the processes that users were involved with, and where exactly the repository should fit. The problem is that research repositories fit at the end of a production process and are therefore providing the service of Archiving. But it is not helpful to archive learning materials. Instead we therefore looked at what other services a teaching and learning repository might provide, finally settling on Hosting (putting materials online easily and with an inline browser preview), Organisation (providing tagging and collection facilities), and Community (giving users a profile page as a way of establishing identity, and emphasizing contribution and remixing).

\section{Case Study 2: Mobile Tools for Placement Learners}

Description: The Mobile Placement Learning and Assessment Toolkit (mPLAT) project aimed to provide a mobile learning toolkit to support practice based learning for nursing students in three different UK Higher Education Institute [15]. Our belief was that practice-based learning would be improved by connecting the student in situ with the competency model they were required to learn. We developed two tools: a Profile Placement Tool that provides guidance for mapping the domain and competencies to the opportunities offered in the placement area, and a Learning Contract Builder that drew on student's experience and a placement profile to create an action plan for learning. We deployed the mPLAT toolkit in three separate trials.

Difficulties and Challenges: We undertook significant preparation for the first trial including setting up training sessions, establishing a regular helpdesk, and getting agreement at all necessary management levels, but despite this many students gave up using the tools within a few weeks, and by the end of the trial there were few users.

Through focus group sessions we heard reports of ward staff demanding that the mobile devices were 'put away', some students were accused of 'texting their friends instead of working'. Students also found the device itself technically challenging, and with the generally negative atmosphere did not feel motivated to overcome the difficulties. This happened even though domain partners were sponsors of the innovation and even though the deployment areas were informed about the trial. 
In our second trial, the sobering experience of our first trial helped us to recognize that co-design for creating useful, innovative tools is not enough to ensure innovation in practice; it requires a method that we are called co-deployment which recognizes the difficulties of deploying tools which may challenge long-held practice, creates initiatives to mitigate them, and brings all stakeholders in the domain community to work together to accept beneficial innovation [16]. In the third trial we also simplified the tools, introducing tagging concepts from web 2.0 to encourage users to link their placement experiences to the competency model. As a result of these changes there was a marked increase in the confidence of the students in the use of the tools, and both students and their mentors found that communication between them improved.

Ergonomic Perspective: An outcome from co-design was that although there was a need for student nurses to become holistic practitioners, there was no pedagogical route in the paper portfolio system for them to achieve it except by reflection on their practice during placements. From a cognitive ergonomics point of view the existing paper system was deficient, giving a clear objective for the technical tool.

Having discovered in the first trial that acceptance of the use of the tools into placements was a significant problem, we found it useful to take a macro-ergonomics perspective, and to look at the cultural norms, practices and environment that students and the nursing professionals were involved with, and where exactly we expected the use of the tools would fit into them. From this perspective, we took forward the principles of co-design to create our co-deployment methodology [16].

Finally, in tackling what we consider as student risk aversion to using novel tools, in the third trial we used a cognitive ergonomics perspective to embed the use of the tool into the processes and activities of the students and practice assessors.

\section{Case Study 3: Framework Reference Model for e-Assessment}

Description: In 2005 the UK Joint Information Systems Committee (JISC) commissioned the authors to run a project called FREMA (the Framework Reference Model for Assessment) to explore the e-assessment landscape and to help the research community find, understand and articulate key challenges [17].

Due to the maturity of the domain we decided that rather than the project team define a number of narrow challenges we would provide an online information wiki where we could guide the community in reporting its own work, and provide analysis tools to help them discover key issues for themselves. However a standard wiki was not powerful enough for our needs. This is because we not only recorded the type of each entry (e.g. was it a project, a piece of software, etc) but we also recorded typed relationships between entries (e.g. this software uses that standard).

As part of this work we also worked closely with the community to develop a topic map of assessment. In the wiki, typed properties allowed us to attach entries to concepts from our topic map, and we then built a flash-based browser that dynamically queried the wiki in order to provide a graphical index into the site.

Difficulties and Challenges: The concept map became a convenient focus for the FREMA community. But it was a challenge to articulate a consistent methodology for 
building the map. For example, we found that one branch of the map might be decomposed functionally while another might be decomposed around artifacts.

Our use of narrative descriptions to capture entries was a success, and we gathered hundreds of examples, but users struggled with wiki syntax, in particular with the complex constructs needed for the Semantic Wiki extensions. We attempted a number of solutions (for example, using templates to make page creation easier, and creating our own SMW extension that acted as a guide that prompted users to complete semantic data and made suggestions, but these were met with limited success [18].

However the biggest difficulty was that we misunderstood community interest in the activity of collecting data and building the concept map, as interest in the tool and analysis itself. Towards the end of the project it became clear that it was the activity that acted as a focus for the community, and when that activity disappeared the community dispersed, making the tool ultimately unsustainable.

Ergonomic Perspective: Building a topic map is a process of knowledge construction. Since we did not enforce any one methodology for this process we were relying on an alignment between the way that individuals thought about the assessment domain, and the way that the concept map requires that to be expressed. This can be considered a problem of cognitive ergonomics, but perhaps more than any other of our examples, it is peculiar to knowledge systems in particular, as it is about the expression of knowledge structures that have grown organically in peoples minds through their own experiences, and the difficultly of asking them to express those in a systematic way that may not match their own frameworks of thought.

Ultimately though, the real difficulty was in our misunderstanding of community interest, a problem of macro-ergonomics. We believed that the challenge that needed to be solved was that people needed to better understand the usage of technology in order to better target their work. In fact we learned that it was collaborative activity of defining the domain (through the topic maps) that was perceived as most valuable.

\section{Conclusions}

We have argued that TEL systems can be considered primarily as knowledge systems, since they are concerned with how knowledge is formed, communicated and applied. Considering knowledge in a system often means focusing on how it is transformed (for example, looking at what users already know, how able they are to express it, how easily it can be interpreted into a machine form, and once there how useful is it).

From our case studies we see evidence of these kinds of transformations (for example, EdShare involved the transformation of tacit knowledge about educational materials into explicit meta-data, and FREMA faced the challenge of transforming the inconsistent and multi-dimensional domain understanding of a community into a single coherent topic map). However we also saw problems concerned with integrating with existing knowledge processes and with how best to use resulting knowledge structures (for example, EdShare had to refocus the services of the repository in order to better fit the requirements and working practices of teachers, and FREMA misunderstood how the community would focus on the activity rather 
than the tool). We therefore arrive at the following general definition of Knowledge System Ergonomics that draws from both cognitive and macro ergonomics:

Knowledge System Ergonomics - the science of building knowledge structures and systems that fit the ways in which the users of those systems conceptualize, express, communicate, process and apply knowledge.

We believe that a good basis for a design framework of Knowledge System Ergonomics would be principles of alignment based on conceptual, cognitive and contextual aspects, to ensure that for each aspect the human, organizational and technical components are aligned. Where misalignment is unavoidable (for example, when knowledge is required at an unusual level of formality that does not naturally occur) processes should be put in place to overcome them (for example, using computational models to bridge the difference, or through appropriate training).

\section{References}

1. Mehanna. e-Pedagogy: the pedagogies of e-learning. ALT-J (2004) vol. 12 (3) pp. 279-293

2. Pittard. Evidence for e-learning policy. Technology, Pedagogy and Education (2004) vol. 13 (2) pp. 181-194

3. Chiu et al. Usability, quality, value and e-learning continuance decisions. Computers \& Education (2005) vol. 45 (4) pp. 399-416

4. Nielsen. Heuristic evaluation. In Usability Inspection Methods (John Wiley \& Sons) (1994)

5. Feldstein. What is usable e-learning?. eLearn (2002) vol. 9 (September)

6. Eason. Ergonomic perspectives on advances in human-computer interaction. Ergonomics (1991) vol. 34 (6) pp. 721-741

7. Hendrick. Future directions in macroergonomics. Ergonomics (1995) v38(8) pp. 1617-1624

8. Falzon. Cognitive ergonomics: understanding, learning and designing human-computer interaction. Cognitive Ergonomics (1990) (ISBN:0-12-248290-5)

9. Smith. The ergonomics of learning: educational design and learning performance. Ergonomics (2007) vol. 50 (10) pp. 1530-1546

10. Rudolf and Griffiths. Evaluating the ergonomics of a student learning environment. Work: A Journal of Prevention, Assessment and Rehabilitation (2009) vol. 34 (4) pp. 475-480

11. Schreiber. Knowledge Engineering and Management: the CommonKADS Methodology. Published by MIT Press (2000) (ISBN: 0-262-19300-0)

12. Davis et al. Bootstrapping a Culture of Sharing to Facilitate Open Educational Resources. IEEE Transactions on Learning Technologies (2010) vol. 3 (1)

13. Millard et al. The Language Box: Re-imagining Teaching and Learning Repositories. Proc of ICALT 2009, Riga, Latvia (2009)

14. Millard et al. Phantom Tasks and Invisible Rubric: The Challenges of Remixing Learning Objects in the Wild. Proc of ECTEL 2009, Nice, France (2009)

15. Zhang et al. A Mobile Toolkit for Placement Learning. Proc of ICALT, Spain (2008)

16. Millard et al. Co-design and Co-deployment Methodologies for Innovative m-Learning Systems. In Multiplatform E-Learning Systems and Technologies: Mobile Devices for Ubiquitous ICT-Based Education, IGI Global. (2009)

17. Millard et al. The e-Learning Assessment Landscape. Proc of ICALT, Netherlands (2006)

18. Kousetti et al. A study of ontology convergence in a semantic wiki. Proc of ACM WikiSym 2008, Porto, Portugal (2008) 